\title{
Enucleación de odontoma compuesto y tracción de un canino superior en un paciente pediátrico
} Reporte de un caso

Andrade Sánchez Eder*, Zaragoza- Velásquez Nicolás**, Ruiz Esparza Romo Claudia Liliana**, Alatorre Pérez Sergio****, González de Santiago Manuel de Jesúss***, Rosas-Cabral Alejandro****.

\section{Resumen}

- Objetivo: Los odontomas representan el 70\% de todos

- los tumores odontógenos, pudiéndose diferenciar dos

- tipos; el complejo y el compuesto, según su morfo-dife-

- renciación. Se presenta un caso de odontoma compues-

- to en un paciente pediátrico con un canino retenido en

- el maxilar. Se presenta el caso de una paciente de

- 12 años de edad asintomática que no ha presentado

- la exfoliación del órgano deciduo 53. La ortopantomo-

- grafía mostró la presencia del OD 13 retenido en el

maxilar, así como la de una tumoración radiopaca con estructuras bien definidas en su interior entre las raíces de los incisivos superiores izquierdos. El estudio histológico del tejido removido fue de odontoma compuesto. Conclusiones: Después de la exéresis de la lesión tumoral y la colocación del botón para tracción canina, se pudo constatar la importancia de métodos diagnósticos más modernos como las tomografias tridimensionales, evitándose errores al momento del abordaje quirúrgico. LUXMÉDICA, AÑO 7 NÚM 21 MAYO-AGOSTO 2012 pp 47-52.

Palabras clave: Tumores odontógenos, odontoma, odontoma compuesto, tracción canina.

\section{Introducción}

Los odontomas son tumores de origen odontogénico no raramente encontrados por los estomatologos ${ }^{1}$ y constituyen el tumor odontogénico más común de la mandíbula en pacientes menores de 20 años. ${ }^{2}$ Los odontomas son las lesiones odontógenas no quísticas

\footnotetext{
* Estudiante de la licenciatura de Médico Estomatólogo. Centro de Ciencias de la Salud. Universidad Autónoma de Aguascalientes.

** Doctor en Ciencias Odontológicas, Profesor Titular del área de Ortodoncia. Centro de Ciencias de la Salud. Universidad Autónoma de Aguascalientes.

*** Profesor Titular del área de Odontopediatría. Centro de Ciencias de la Salud. Universidad Autónoma de Aguascalientes.

**** Profesor Titular del área de Cirugía Oral. Centenario Hospital Miguel Hidalgo. Universidad Autónoma de Aguascalientes.

***** Profesor investigador del Departamento de Medicina. Centro de Ciencias de la Salud Universidad Autónoma de Aguascalientes.
}

Fecha de recibido: 20 de marzo 2012

Fecha de aceptación: 29 de junio 2012

Correspondencia: Dr Nicolás Zaragoza Velázquez. Profesor investigador del Departamento de Estomatología del Centro de Ciencias de la Salud de la Universidad Autónoma de Aguascalientes. Avenida Universidad 940, CP 20131, Cuidad Universitaria. Aguascalientes, Ags., México. Teléfono 014499107400 . Correo electrónico 
más frecuentes y representan casi el $70 \%$ de todos los tumores odontógenos. Se reconocen dos tipos histológicos de los mismos: odontoma complejo y compuesto. Los odontomas compuestos son malformaciones que involucran todos los tejidos dentales normales y tienen numerosas estructuras similares a dientes (con alteraciones en su forma y tamaño) llamados dentículas, ${ }^{3}$ en tanto que los odontomas complejos son tumores bien formados con tejidos dentales en una distribución desorganizada. ${ }^{4}$ Se cree que esta anomalía se presenta durante las etapas de iniciación y proliferación y a menudo alcanzan un tamaño fijo, no se consideran neoplasias verdaderas, sino hamartomas. ${ }^{5}$ Casi todos se presentan en pacientes que están en la primera y segunda década de la vida. Los odontomas aparecen con más frecuencia en el maxilar que en la mandíbula, las lesiones suelen descubrirse por el retardo en la erupción de un diente permanente en el tiempo adecuado, casi siempre con el retraso en la exfoliación del diente deciduo. ${ }^{6}$ Los odontomas compuestos suelen estar localizados en la parte anterior de la boca, sea sobre las coronas de dientes no erupcionados, o bien, entre las raíces de los erupcionados. El odontoma compuesto contiene estructuras radiopacas múltiples que se parecen a dientes en miniatura, estos odontomas compuestos pueden contener pocas (2-3) o muchas (de 20-30) estructuras análogas a dientes en miniatura, en contraparte el odontoma complejo se caracteriza por ser un masa nudosa sólida en la cual no se puede identificar macroscópicamente la forma de un diente, ${ }^{7}$ el diagnóstico de los mismos habitualmente se establece mediante estudios radiográficos de rutina, debido a que usualmente son asintomáticos, y se presentan como tumores bien circunscritos con áreas radiopacas y radiolúcidas que semejan dientes, dependiendo del tipo y grado de calcificación del mismo. ${ }^{8}$ El diagnóstico diferencial de los odontomas incluye otros tumores odontogénicos como son el fibroma ameloblástico, el fibroodontoma y el odontoameloblastoma. ${ }^{4}$

En el presente trabajo nosotros describimos el caso de un paciente pediátrico con un odontoma compuesto, en el que se emplearon nuevas tecnologías para su diagnóstico.

\section{Presentación del caso}

Paciente femenino de 12 años de edad, que se remitió a la clínica de cirugía por presentar una lesión radiopaca con estructuras similares a dientes en su interior, a nivel del órgano deciduo (OD) 22, así como también el OD 13 retenido en el maxilar (fig. 1). La paciente no presentaba antecedentes personales de interés y su arcada superior presentaba el OD 53.

Durante la exploración clínica se observó la presencia del OD 53 en oclusión, mediante la palpación en la región anterior del paladar se detectaron dos abulta- 
mientos que correspondían al canino retenido y a la estructura del odontoma (fig. 2). Por tal motivo se solicitó una ortopantomografía y una tomografía computarizada tridimensional (fig. 3) (fig. 4). En las imágenes tridimensionales de la tomografía se verificó que la ubicación del odontoma compuesto se encontraba a $2 \mathrm{~mm}$ del incisivo lateral superior izquierdo, dicha estructura tumoral no había causado desplazamiento ni obstrucción de algún órgano dental. Bajo el diagnóstico presuntivo de odontoma compuesto se realizó el plan de tratamiento que consistió en la enucleación del mismo, la exéresis de la tumoración, la exodoncia del OD 53, así como la exposición y colocación de un botón para tracción ortodóncica del OD 13 (fig. 5). Posteriormente se realizó estudio histopatológico del tejido removido confirmándose el diagnóstico de odontoma compuesto (fig. 6). Después de seis meses de la intervención quirúrgica, el paciente permaneció libre de sintomatología, sin complicaciones y se inició el tratamiento ortodóntico correctivo.

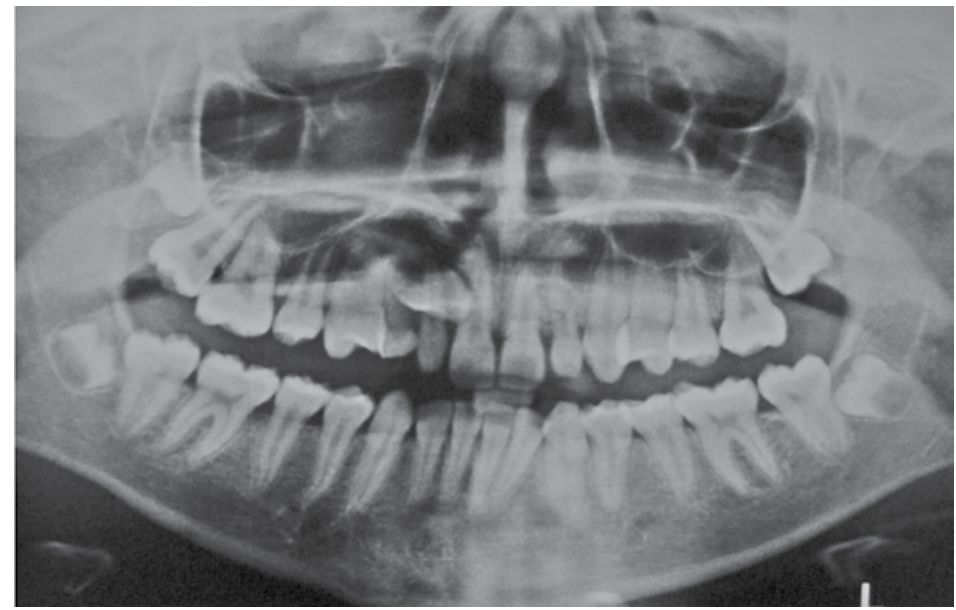

Fig. 1.- Ortopantomografía que muestra el O.D. 13 retenido y la lesión radiopaca a nivel del O.D. 22.

Fig. 2.- Aspecto inicial del maxilar; Nótese las prominentes áreas clínicas, tanto del odontoma como del canino retenido, en la parte anterior.

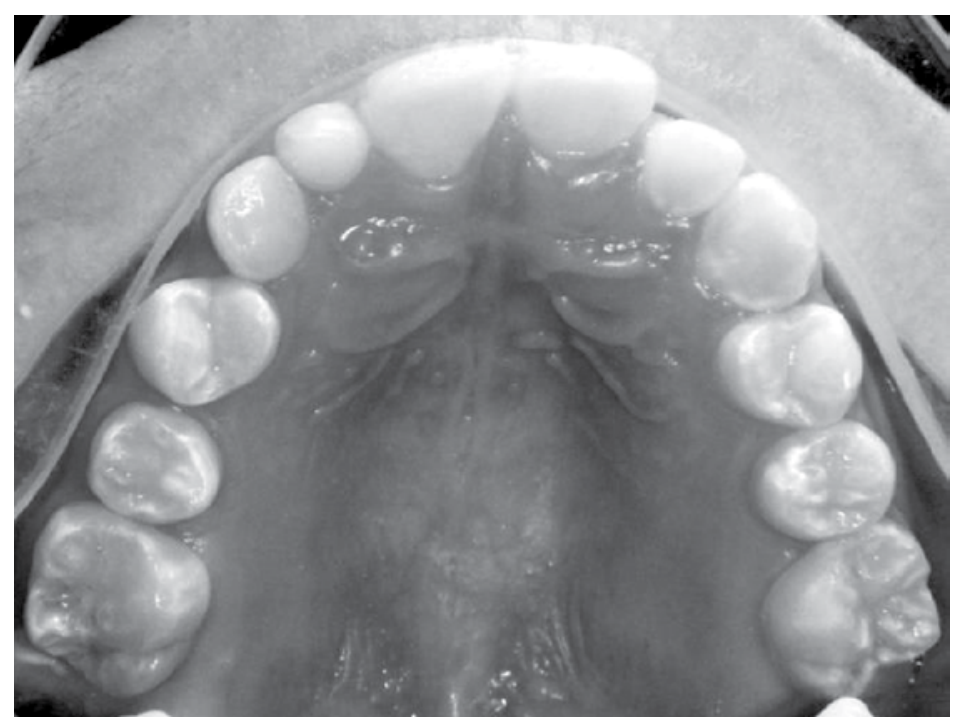




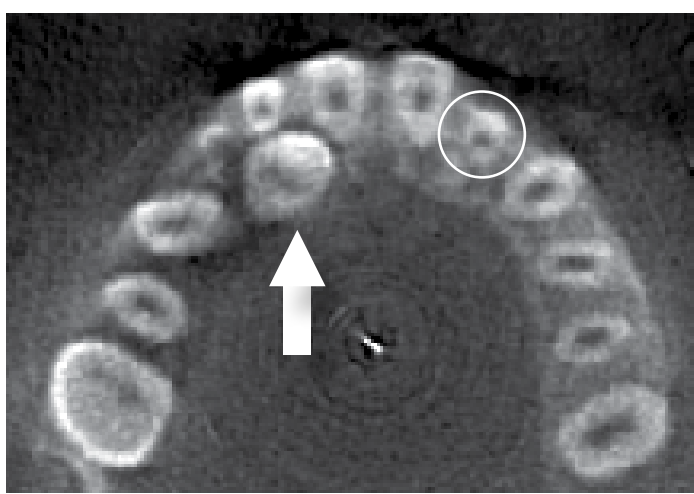

Fig. 3.- Tomografía computarizada que muestra la localización exacta del canino (flecha) y del odontoma compuesto (círculo).

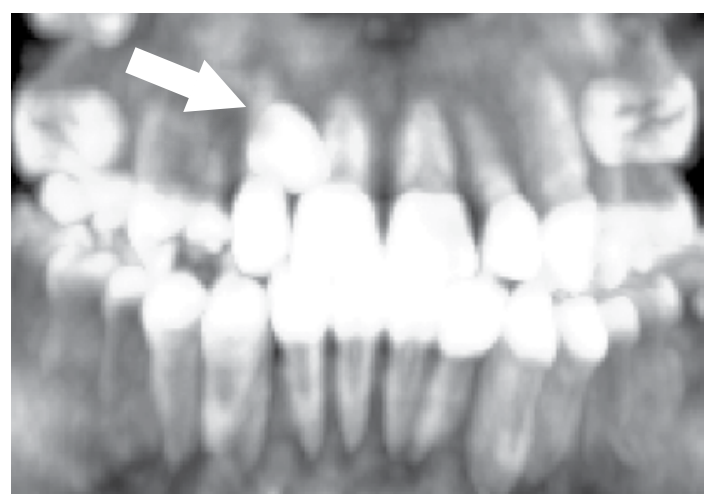

Fig. 4.- Tomografía tridimensional que muestra el canino retenido en el maxilar.

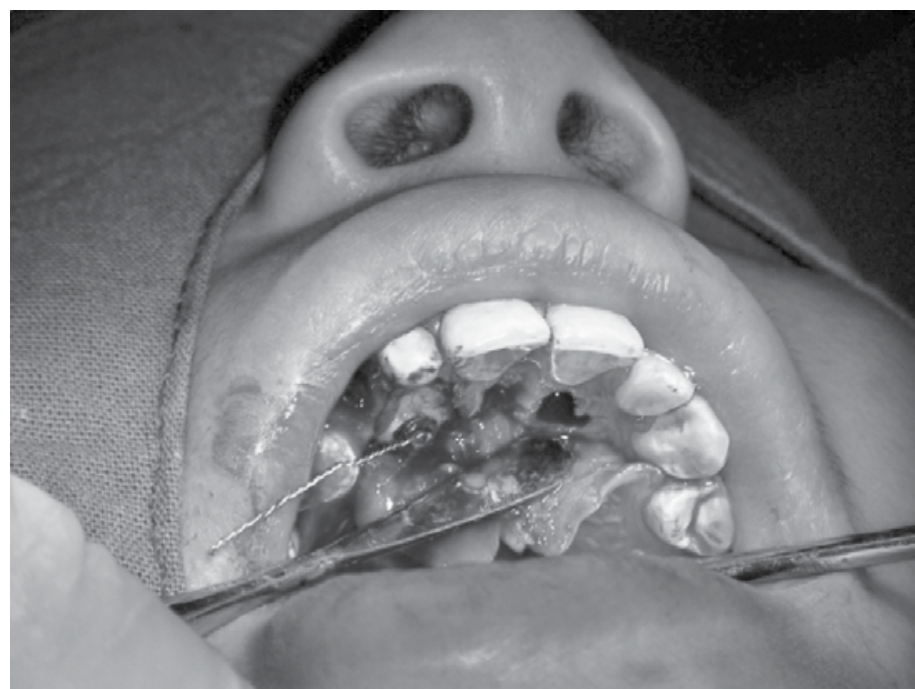

Fig. 5.- Fotografía transoperatoria que muestra el sitio de exéresis del odontoma, y la corona del OD 13 con botón para tracción.

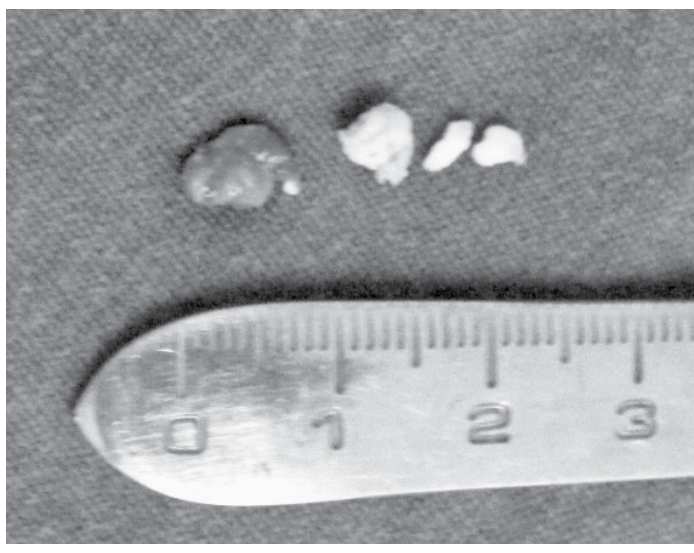

Fig. 6.1.- Aspecto macroscópico de las piezas quirúrgicas (saco folicular y tejido mineralizado).

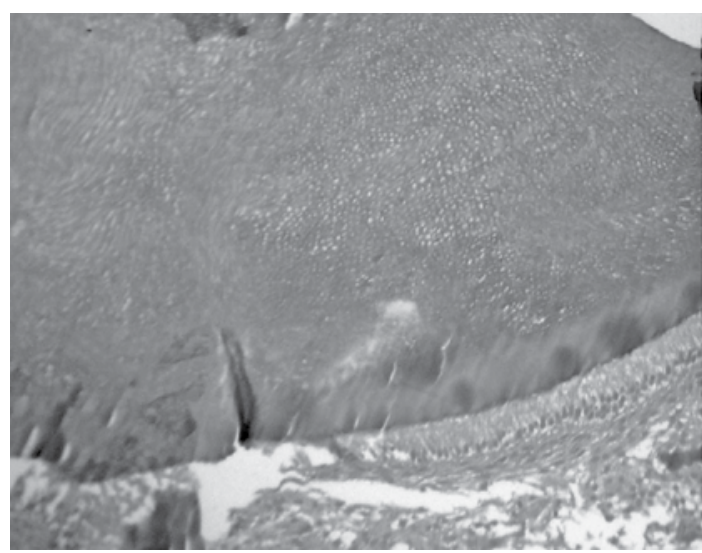

Fig. 6.2.- Aspecto microscópico del tejido mineralizado que muestra túbulos dentinarios y capa de ameloblastos 


\section{Discusión}

Aunque se ha reportado que los odontomas se pueden encontrar a cualquier edad, en el presente, como en la mayoría de los reportes de la literatura, describimos un caso de un paciente de la segunda década de la vida ${ }^{4}$, así mismo su localización en la región anterior del maxilar es de las más frecuentemente reportadas, con un curso clínico asintomático y que como lo refieren otros investigadores, su diagnóstico fue orientado por el retraso en la erupción del diente deciduo correspondiente ${ }^{9}$. Cabe destacar que aunque la mayor parte de estos pacientes se diagnostican mediante radiografías periapicales, en nuestro caso fue realizado mediante una radiografía panorámica y se complementó el mismo mediante la realización de una tomografía tridimensional, lo cual es acorde a las recomendaciones dadas por otros autores ${ }^{2}$. Así mismo, de acuerdo a lo recomendado por diversos autores la remoción quirúrgica del odontoma es el tratamiento de elección, debido a que en la mayoría de los casos los mismos se encuentran bien delimitados, lo que facilita su exéresis, como fue en nuestro caso. Como en la mayoría de los odontomas, en este trabajo el estudio histopatológico reveló un odontoma compuesto, en concordancia con lo reportado en la literatura ${ }^{10}$. Es conveniente enfatizar la importancia que tiene, ante un paciente pediátrico, analizar a fondo su historial clínico, ya que es el medio en el que el médico estomatólogo puede detectar alguna anomalía hereditaria de órganos dentales retenidos o agenesias. El control del dolor es un aspecto de cuidado en este caso, ya que, con el hecho de tratarse de un paciente pediátrico, los protocolos a seguir tendrán que ser más eficientes, tanto en el caso del manejo conductual, como en el procedimiento propiamente dicho. El empleo de técnicas diagnósticas mediante tomografia fue necesario en este caso clínico para facilitar el abordaje quirúrgico y de esta forma tener la certeza de que se tendrá acceso a los sitios en los que se localizan las patologías intraóseas. Este tipo de casos clínicos, requieren opiniones interdisciplinares, por tal razón se llevo a cabo bajo la supervisión cuidadosa de los respectivos especialistas, para el adecuado manejo del paciente; el ámbito de odontopediatría, ortodoncia y cirugía maxilofacial con el fin de darle un adecuado seguimiento postratamiento.

\section{Conclusiones}

Los odontomas son los tumores odontógenos más comunes que pueden presentarse en la práctica clínica, debido a que son asintomáticos se hace necesaria una exploración radiográfica para su oportuna detección. Por otra parte el uso de tomografía computarizada tridimensional fue una ventaja extra para facilitar el tratamiento en este caso.

En el presente caso clínico se vio la necesidad de realizar interconsultas de varias especialidades como odontopediatría, ortodoncia y cirugía maxilofacial con el fin de efectuar un tratamiento apropiado para el paciente, finalmente se deberá proceder al control y seguimiento del caso clínico para asegurar el éxito del tratamiento a largo plazo. 


\section{Bibliografía}

1.- Boffano P, Zavattero E, Roccia F, Gallesio C. Complex and compound odontomas. J Craniofac Surg. 2012;23(3):685-688.

2.- Iatrou I, Vardas E, Theologie-Lygidakis, Leventis $M$. A retrsopective analysis of the chraacteristics, treatment and follow-up of 26 odontomas in Greek children. J of Oral Sci. 2010;52(3):439-447.

3.- López-Areal L, Silvestre Donat F, Gil-Lozano J. Compound odontoma eruptimg in the mouth: 4 year follow-up of a clinical case. J Oral Pathol med. 1992;21:285-288.

4.- Hidalgo-Sánchez O, Leo-Berrocal MI, MartínezGonzález JM. Metaanalysis of the epidemiology and clinical manifestations of odontomas. Med oral $\mathrm{Pa}$ thol Oral Cir Bucal. 2008;13:E730-E734.

5.- Sapp J. Philip. Patología oral y maxilofacial contemporánea. Segunda edición. Barcelona, España: Editorial Elsevier, pags. 155-160.

6.- Tomizawa M, Osuka Y, Noda T. Clinical observations of odontomas in Japanese children: 39 cases including one recurrent case. InT J Paediatric Dent. 2005; 15:37-43.
7.- Tekkesin MS, Pehilvan S, Olgac V, Aksakalli N, Alatli C. Clinical and histopathological investigation of odontomas: Review of the literatura and presentation of 160 cases. J Oral and Maxillofacial Surgery. 2012;70(6);1358-1361.

8.- Amado Cuesta S, gargallo AJ, Berini Aytés L, Gay Escoda C. review of 61 cases of odontoma. Presentation of an erupted complex odontoma. Med Oral 2003;8:366-373.

9.- Serra-Serra G, Berini-Aytés L, Gay-Escoda C. Erupted odontomas: a report of three cases and review of the literatura. Med Oral Pathol Oral Cir Bucal. 2009;14: E299-E303.

10. Buchner A, Merrel PW, Carpenter WM. Relative frequency of central odontogenic tumors: a study of 1088 cases from Nothern california and comparison to studies from other parts of the world. J Oral MaxiIlofac Surg. 2006;64:1343-1352. 